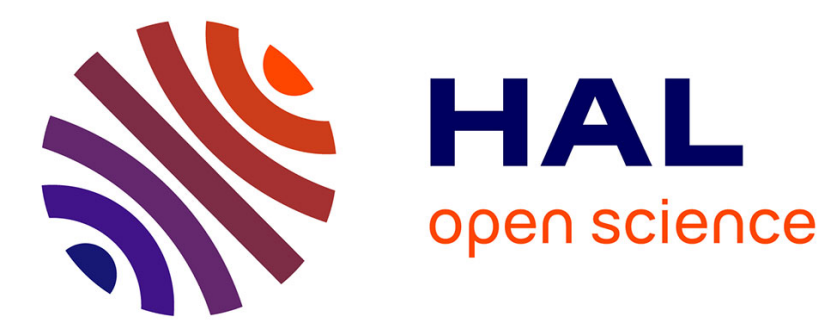

\title{
Muscle Strength and Mass Distribution Identification Toward Subject-Specific Musculoskeletal Modeling
}

Mitsuhiro Hayashibe, Gentiane Venture, Ko Ayusawa, Yoshihiko Nakamura

\section{To cite this version:}

Mitsuhiro Hayashibe, Gentiane Venture, Ko Ayusawa, Yoshihiko Nakamura. Muscle Strength and Mass Distribution Identification Toward Subject-Specific Musculoskeletal Modeling. IROS: Intelligent RObots and Systems, Sep 2011, San Francisco, United States. pp.3701-3707, 10.1109/IROS.2011.6094863 . lirmm-00637754

\section{HAL Id: lirmm-00637754 https://hal-lirmm.ccsd.cnrs.fr/lirmm-00637754}

Submitted on 2 Nov 2011

HAL is a multi-disciplinary open access archive for the deposit and dissemination of scientific research documents, whether they are published or not. The documents may come from teaching and research institutions in France or abroad, or from public or private research centers.
L'archive ouverte pluridisciplinaire HAL, est destinée au dépôt et à la diffusion de documents scientifiques de niveau recherche, publiés ou non, émanant des établissements d'enseignement et de recherche français ou étrangers, des laboratoires publics ou privés. 


\title{
Muscle Strength and Mass Distribution Identification Toward Subject-Specific Musculoskeletal Modeling
}

\author{
Mitsuhiro Hayashibe, Gentiane Venture, Ko Ayusawa, Yoshihiko Nakamura
}

\begin{abstract}
In current biomechanics approach, the assumptions are commonly used in body-segment parameters and muscle strength parameters due to the difficulty in accessing those subject-specific values. Especially in the rehabilitation and sports science where each subject can easily have quite different anthropometry and muscle condition due to disease, age or training history, it would be important to identify those parameters to take benefits correctly from the recent advances in computational musculoskeletal modeling. In this paper, Mass Distribution Identification to improve the joint torque estimation and Muscle Strength Identification to improve the muscle force estimation were performed combined with previously proposed methods in muscle tension optimization. This first result highlights that the reliable muscle force estimation could be extracted after these identifications. The proposed framework toward subject-specific musculoskeletal modeling would contribute to a patient-oriented computational rehabilitation.
\end{abstract}

\section{INTRODUCTION}

\section{A. Background}

An understanding of the whole body human motion is a complex process that requires information at different scales. The observation of a motion reflects in fact the brain activity, the neural system activity, and the musculo-skeletal system activity. To fully understand the mechanisms and the internal network of human motion generation, it is thus mandatory to understand each element and the connections between these elements in a multidisciplinary framework. Muscles are the basic elements to actuate the joints in the human body [1]. Analyzing how they activate and generate forces for a specific motion would significantly improve several research areas ranging from physical therapy to neuro-rehabilitation. In addition, quantitative analysis between neural activity and muscle force can contribute to the design of Functional Electrical Stimulation (FES) for paralyzed muscles and myoelectrical limb control, and also to the design of robotic prosthetic limbs and exoskeletons.

The modeling of the human body and the computation of human dynamics is an active field in robotics and biomechanics. The general musculoskeletal model of whole body and its dynamics computation method were established and

Authors acknowledge the support provided by the INRIA-JSPS joint program AYAME (2010-2012).

M. Hayashibe is with INRIA Sophia Antipolis -DEMAR Project and LIRMM, UMR5506 CNRS UM2, 161 Rue Ada, 34095 Montpellier, France hayashibedlirmm. fr

G. Venture is with Department of Mechanical Systems Engineering, Tokyo University of Agriculture and Technology, 2-24-16 Nakacho, Koganei, 184-8588 Tokyo, Japan venture@cc.tuat.ac.jp

K. Ayusawa and Y. Nakamura are with Department of MechanoInformatics, University of Tokyo, 7-3-1 Hongo, Bunkyo-ku, 113-8656 Tokyo, Japan ayusawa, nakamura@ynl.t.u-tokyo.ac.jp are now available as OpenSim software [2][3]. The efficient computational algorithms for musculoskeletal model were pursued for inverse and forward dynamics on the basis of efficient multibody dynamics computations [4][5].

In this paper, we discuss the muscle strength and mass distribution identification toward subject-specific musculoskeletal modeling. As mentioned above, many researches on musculoskeletal modeling have been reported especially in the biomechanics society. These techniques are getting mature to be applied for the clinical rehabilitation study and other specific purposes. However, to our knowledge, most of the works use the literature-based anthropometric index for segment mass distribution. The work of de Leva [6] which adjusted the Zatsiorsky model is commonly used for segment definitions to obtain "typical" mass distribution. The subject weight, height are used as the reference to compute the corresponding mass of each segment derived from anthropometric table. As it depends on the total mass, it should not be able to represent the subject-specific difference of mass distribution in the case where the subjects have the same weights and heights, the one is an athlete, and the other is an elderly person. In addition, regarding muscle isometric force table, the work of Delp [3] is quite famous and often used when the muscular force is computed based on electromyography (EMG). This musculotendon parameters database is meaningful as it is normally difficult to access these typical values. However, we can not assume that every subject would have similar muscle strength especially in the rehabilitation and sports science where each subject has quite different muscle condition due to disease, age or training history. In fact, the group even in the same generation and healthy people can easily have different muscle strength depending on daily exercises and the preference of sports.

\section{B. Related works}

Inverse dynamics of musculoskeletal models is commonly used to estimate the joint torques and muscle tensions from motion, ground contact force, and/or EMG measurements. Known problems with the inverse dynamics approach is that one must know the inertia and mass of each body segment in order to estimate joint moments correctly [7]. Typically these are estimated using values from cadavers and scaled using simplistic scaling rules, the accuracies of which are rarely verified. This scaling technique is employed also in OpenSim [3]. A novel identification method was proposed and implemented for the human body-segment mass-parameters [8]. This method can contribute to reduce 
errors in the inverse dynamics result significantly. Its realtime implementation is also available [9].

The resultant joint torques from inverse dynamics are net values contributed from every muscle. As the numbers of muscles span for each joint is greater than the degrees of freedom for each joint, the estimation of each muscle force is a redundant problem. Thus, usage of EMG has been studied by many researchers in biomechanics. EMG-based model relies on measured muscle activity to estimate muscle force. Potvin et al. [13] derived the relationship between the EMG signal and muscle force to estimate the muscle load during lifting motion. EMG-based models have been used to estimate torques around the knee joint by calibrating the muscle parameters for individual subjects [10]. However, previously proposed methods for the estimation of forces based on EMG with calibration were about only one DOF as it requires dynamometer to obtain reliable joint torque. Recently, EMG-driven musculoskeletal model was proposed for multiple DOFs of the joints [16]. This method uses Opensim to obtain joint torques from motion capture data.

Drawback of complete EMG-driven musculoskeletal model is that it is unrealistic to access all the inner muscles. In addition, the tensions computed from EMG data are not reliable due to the noise in EMG measurements and uncertain muscle model parameters. Thus, Yamane and Nakamura proposed to obtain physically and physiologically reasonable whole-body muscle tensions by forming an optimization problem such that the error of the tension-torque transformation equation becomes minimum while respecting the physiological muscle dynamics and the muscle activity when it is available from EMG [5]. All inner muscles are included in the optimization. However, subject-specific muscle strength is not considered yet. Identification method to identify subject-specific muscle states and maximal force parameter was developed for FES in [15]. In biomechanics, normally dynamometer is used for subject-specific muscle strength information.

This work is the first trial to make the identification on subject-specific muscle strength and mass distribution based on motion capture. The concept of this paper is summarized in Fig. 1. The white colored parts are the standard processes in the current musculoskeletal analysis. As the subjectspecific mass distribution is identified using kinematic information and ground reaction force, the computed joint torques from the inverse dynamics of musculoskeletal models can be improved to output reliable physically consistent torques. In addition, as the subject-specific muscle strength is identified with the above torque and EMG signal, the optimization result of muscle tensions can be improved to output reliable physiologically consistent torques reflecting subject-specificity.

\section{MASS-DISTRIBUTION IDENTIFICATION}

The equations of motions of a moving system can be written as Eq. 1 [17]. The upper equation describes the 6 DOF motion of a free link: the base-link in the 3D space. The lower equation describes the motion of the various

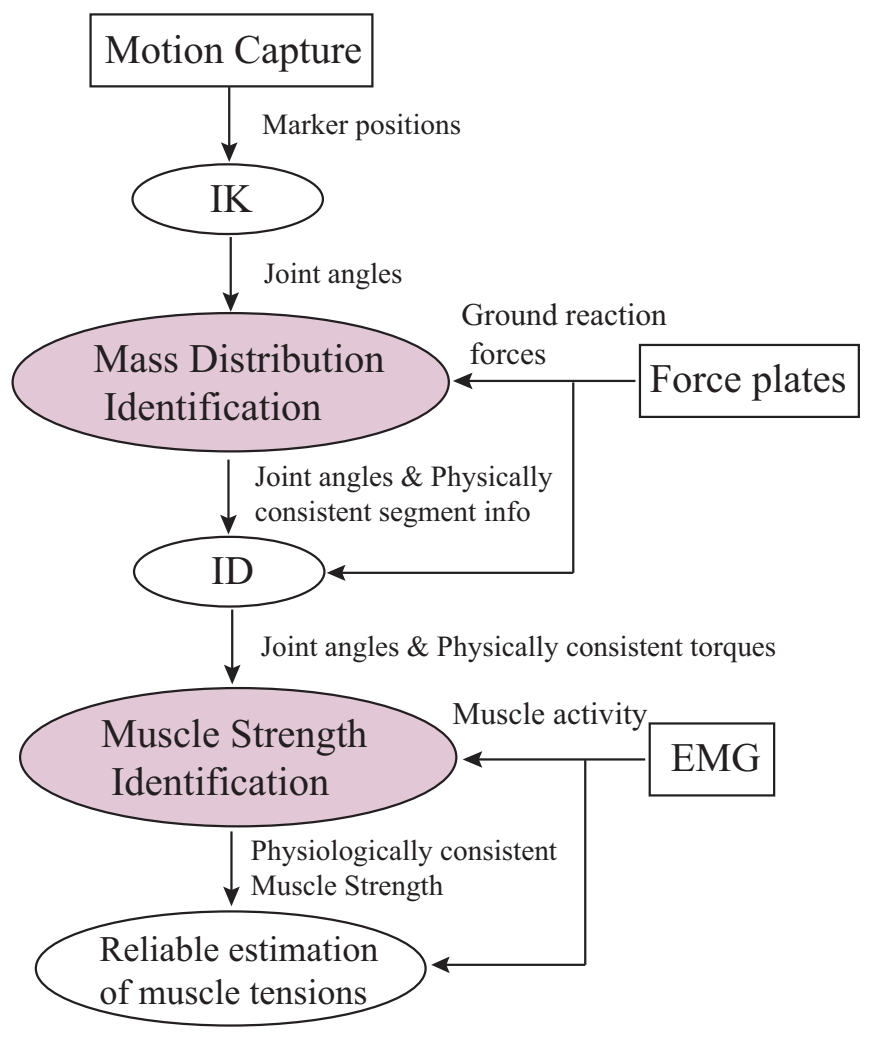

Fig. 1. Scheme of Mass Distribution and Muscle Strength identification.

kinematic chains constituting the whole system. The baselink can be chosen arbitrarily, however for practical reasons it is convenient to select the lower torso. The chains are formed with the limbs, the upper-torso, the head.

$$
\left[\begin{array}{ll}
\boldsymbol{H}_{o o} & \boldsymbol{H}_{o c} \\
\boldsymbol{H}_{c o} & \boldsymbol{H}_{c c}
\end{array}\right]\left[\begin{array}{c}
\ddot{\boldsymbol{q}}_{o} \\
\ddot{\boldsymbol{\theta}}
\end{array}\right]+\left[\begin{array}{l}
\boldsymbol{b}_{o} \\
\boldsymbol{b}_{c}
\end{array}\right]=\left[\begin{array}{l}
\mathbf{0} \\
\boldsymbol{\tau}
\end{array}\right]+\sum_{k=1}^{N_{c}}\left[\begin{array}{l}
\boldsymbol{K}_{k o} \\
\boldsymbol{K}_{k c}
\end{array}\right] \boldsymbol{F}_{k}
$$

where:

- $\boldsymbol{H}_{i j}(i, j=o, c)$ is the inertia matrix,

- $\boldsymbol{q}_{\boldsymbol{o}}$ is the vector of generalized coordinates which represents the 6 DOF of the base-link,

- $\boldsymbol{\theta}$ is the vector joint angles,

- $\boldsymbol{b}_{i}$ is the bias force vector including centrifugal, Coriolis and gravity forces,

- $\tau$ is the vector of joint torques,

- $N_{c}$ is the number of contact point with the environment,

- $\boldsymbol{F}_{k}$ is the $k^{t h}$ vector of external forces,

- $\boldsymbol{K}_{k o}$ and $\boldsymbol{K}_{k c}$ are matrices which map $\boldsymbol{F}_{k}$ to the generalized force vector.

In the aim of identification of the dynamics it is common to rewrite Eq.1 by separating the time-constant inertial parameters $\phi$ from the time varying functions $\boldsymbol{Y}$ [18], resulting in the identification model given by Eq.2. We have shown in our previous work [8] that it is possible to solve the identification problem using solely the upper equation of Eq.2, thus without requiring the joint torque information. This method is particularly adequate for the measurement of the human body dynamics. 


$$
\left[\begin{array}{l}
\boldsymbol{Y}_{o} \\
\boldsymbol{Y}_{c}
\end{array}\right] \boldsymbol{\phi}=\left[\begin{array}{l}
\mathbf{0} \\
\boldsymbol{\tau}
\end{array}\right]+\sum_{k=1}^{N_{c}}\left[\begin{array}{l}
\boldsymbol{K}_{k o} \\
\boldsymbol{K}_{k c}
\end{array}\right] \boldsymbol{F}_{k}
$$

where:

- $\boldsymbol{Y}=\left[\begin{array}{l}\boldsymbol{Y}_{o} \\ \boldsymbol{Y}_{c}\end{array}\right]$ is the regressor, which is function of the system joint angles $\boldsymbol{\theta}$, velocity $\dot{\boldsymbol{\theta}}$ and acceleration $\ddot{\boldsymbol{\theta}}$, and of the vector of generalized coordinates $\boldsymbol{q}_{o}$ and its derivatives;

- $\phi$ is the vector of inertial parameters to estimate such that:

$$
\phi=\left[\begin{array}{llll}
\phi_{0}^{T} & \phi_{1}^{T} & \cdots & \phi_{N}^{T}
\end{array}\right]^{T}
$$

- $\phi_{j}$ is the vector of standard parameters for each link $B_{j}(j=0$ to $N)$, such that:

$$
\phi_{j}=\left[\begin{array}{cccccc}
m_{i} & m s_{i, x} & m s_{i, y} & m s_{i, z} & I_{i, x x} & \\
& I_{i, y y} & I_{i, z z} & I_{i, y z} & I_{i, z x} & I_{i, x y}
\end{array}\right]^{T}
$$

- $m_{j}$ is the mass,

- $I_{j, x x}, I_{j, y y}, I_{j, z z}, I_{j, y z}, I_{j, z x}, I_{j, x y}$ are the 6 independent components of the inertia matrix $\boldsymbol{I}_{j}$,

- $m s_{i, x}, m s_{i, y}, m s_{i, z}$ are the first moments components of the vector $\boldsymbol{m} \boldsymbol{s}_{j}$

Because the measuring environment is not free from noise and measuring errors, and the modeling of the human body is an approximation, the identification results are biased. This bias, even with sufficiently exciting trajectories may lead to parameters that are not physically consistent. Namely, inertia matrices should be definite positive, masses should positives, and the center of a mass of a link appropriately located in the link. In order to guarantee the physical consistency of each parameters we have also included a constraint based on a simplified shape of the link [9]. For the geometric identification: lengths of limbs, center of joints, we use the marker positions captured from a T-pose to generate automatically the adequate model.

The model used for segment identification and the musculo-skeletal model differ in the number of degrees of freedom. This is because for identification we must consider the identifiability problem, and for the musculoskeletal we must take into account via-points of muscles. In the musculo-skeletal model several virtual links or fixed DOF are utilized, and the trunk is divided in links of small size. The identified parameters must then be adjusted to this model. The limbs, the head are globally the same, so we use directly the identified parameters. For the other links, we use a proportional distribution of mass and inertia to reflect the results of identification, while preserving the high number of DOF of the musculo-skeletal model.

\section{TORQUE TO FORCE MAPPING IN IDENTIFIED MODEL}

From the lower part of Eq. 1, once subject-specific inertia matrix is identified, more reliable joint torques $\tau$ can be computed with external forces using Newton-Euler inverse dynamics as in Eq.5. This process is indicated as ID in Fig. 1.

$$
\boldsymbol{\tau}=\left[\boldsymbol{H}_{c o} \boldsymbol{H}_{c c}\right] \ddot{\boldsymbol{\theta}}+\boldsymbol{b}_{c}-\sum_{k=1}^{N_{c}} \boldsymbol{K}_{k c} \boldsymbol{F}_{k}
$$

To obtain the relationship between the joint torques $\tau$ and the muscle element tensions $f$, the Jacobian matrix of the element lengths with respect to the generalized coordinates $\boldsymbol{J} \in \boldsymbol{R}^{n_{m u s} \times n_{\text {dof }}}$ is computed as Eq. 6 .

$$
\boldsymbol{J}=\frac{\partial \boldsymbol{l}}{\partial \boldsymbol{\theta}_{G}}
$$

where:

- $\boldsymbol{l} \in \boldsymbol{R}^{n_{m u s}}$ is the muscle lengths,

- $\boldsymbol{\theta}_{G} \in \boldsymbol{R}^{n_{\text {dof }}}$ is the generalized coordinate,

- $n_{\text {mus }}$ and $n_{d o f}$ are the number of muscles and DOF of the model.

The method for computing $\boldsymbol{J}$ is detailed in [4]. The principle of virtual works yield the following equation relating the muscle forces and the joint torques.

$$
\boldsymbol{\tau}=\boldsymbol{J}^{T} \boldsymbol{f}
$$

Solving Eq.7 for muscle forces $f$ using joint torques $\tau$ is a highly redundant problem. Because the number of muscles $n_{\text {mus }}$ is greater than the total DOF $n_{\text {dof }}$. The skeletal model used in this work has 83 DOF with 314 muscles.

\section{EMG PROCESSING AND MUSCLE CONTRACTION DYNAMICS}

The transformation from EMG to muscle activation is an essential process in Hill-type muscle models [11][12], it is dominant process because the estimated muscle force is assumed to be proportional to the muscle activation. One can refer to the detailed steps in [5]. First, mean of the raw EMG was taken to offset the baseline of EMG signal. The integrated EMG signal, called IEMG, normalized with the value of Maximum Voluntary Contraction (MVC) gives the activity level $u$. The muscle activity $a$ is obtained by the following first-order differential equation:

$$
\begin{aligned}
\dot{a}=\frac{u-a}{T} & T & =T_{a}(u \geq a) \\
& T & =T_{d}(u<a)
\end{aligned}
$$

where $T_{a}$ and $T_{d}$ are the time constants for activation and deactivation.

The Hill-type model is used to estimate the reference muscle force $f^{*}$ with general form of the function by

$$
f^{*}=a f_{l}(l) f_{v}(i) F_{\text {iso }}
$$

where:

- $F_{i s o}$ is the maximum isometric muscle force,

- $l$ is the muscle length,

- $f_{l}(l)$ and $f_{v}(i)$ gives the normalized force-length and force-velocity relationship respectively [7].

The force length relationship shows a Gaussian distribution around the optimal length $l_{0}$ and is formulated as

$$
f_{l}(l)=\exp \left\{-\left(\frac{l-l_{0}}{K_{l}}\right)^{2}\right\}
$$


where $K_{l}$ is a constant parameter.

$f_{v}(i)$ represents the relationship between velocity and normalized force. The muscle can contract at its maximum velocity $v_{\max }$ without load and slows down as the load increases. This relationship is formulated as follows:

$$
f_{v}(i)=\left\{\begin{array}{cc}
0 & \left(i \leq-v_{\max }\right) \\
\frac{V_{\text {sh }}\left(v_{\max }+i\right)}{V_{s h} v_{\max }-i} & \left(-v_{\max } \leq i \leq 0\right) \\
\frac{\left.V_{s h} V_{\text {shl }} v_{\max }+V_{\operatorname{mi}} i\right)}{V_{s h} V_{\text {shl }} v_{\max }+i} & (0 \leq i)
\end{array}\right.
$$

where $V_{s h}, V_{s h l}$ and $V_{m l}$ are constant parameters given by Stroeve [14].

\section{MUSCLE STRENGTH IDENTIFICATION}

Considering the joint torque $\tau_{k}$ which is generated by the muscle $k$,

$$
\begin{aligned}
\boldsymbol{\tau}_{k} & =\boldsymbol{J}_{k}^{T} f_{k} \\
& =\left[-a_{k} f_{l_{k}}\left(l_{k}\right) f_{v_{k}}\left(i_{k}\right) \boldsymbol{J}_{k}^{T}\right] F_{i s o k} \\
& =\boldsymbol{C}_{k}^{T} F_{i s o k}
\end{aligned}
$$

where $\boldsymbol{J}_{k} \in \boldsymbol{R}^{1 \times n_{d o f}}$ is the element Jacobian for muscle $k$. As $f_{l_{k}}\left(l_{k}\right), f_{v_{k}}\left(i_{k}\right), \boldsymbol{J}_{k}^{T}$ can be obtained from the result of inverse kinematics and $a_{k}$ is obtained from EMG measurement for the muscles with EMG electrodes, $\boldsymbol{C}_{k}$ is known for the muscles with EMG electrodes. We call $C_{k}$ as net contribution matrix.

As the human motion is generated by complex combination of multiple muscle forces, then it is not always the case but for certain tasks, particular joint torque among $\tau$ is expected being consisted of the contributions from particular muscles. In such case and with the assumption where synergist muscles exhibit similar activity levels like the muscle grouping in stretch reflex, the net contribution matrix $C_{k}$ are available if there is EMG measurement in the muscle group, certain elements in joint torque vector can be expressed as follows:

$$
\begin{aligned}
\boldsymbol{\tau}(j) & =\sum_{k=1} \boldsymbol{C}_{k}^{T}(j) F_{i s o k} \\
& =\sum_{G_{e m g}} \boldsymbol{C}_{k}^{T}(j) F_{i s o k}+d
\end{aligned}
$$

where:

- $(j)$ represents $j$ th element of the vector,

- $G_{e m g}$ is the EMG measured synergist muscle group,

- $d$ is the constant residual term which takes into account the contribution from other elements.

The subject-specific Muscle-Strength $F_{i s o k}$ is identified with linear regression.

After the identification of subject-specific Muscle-Strength $F_{i s o k}$, a more reliable EMG-based reference muscle force $f^{*}$ can be computed. From the previous work [4] about optimization criteria for the actuation redundancy problem in quadratic programming, we should now minimize the objective function to find a solution $\boldsymbol{f}$ keeping the error against EMG-based muscle tension which is physiologically consistent and the error against the resultant joint torque which is physically consistent and subject-specific. The optimization is formulated as follows:

Find $f$ that minimizes

$$
Z=\frac{1}{2}\left|\boldsymbol{\tau}-\boldsymbol{J}^{T} \boldsymbol{f}\right|^{2}+\frac{W_{e m g}}{2}\left|\boldsymbol{f}-\boldsymbol{f}^{*}\right|^{2}
$$

subject to the following constraints:

$$
\boldsymbol{E}_{m} \boldsymbol{f} \leq 0
$$

where $W_{e m g}$ is the weight for EMG-based force reliability coefficient and $\boldsymbol{E}_{m}$ is a matrix which extracts the tensions of all elements from $\boldsymbol{f}$.

Defining the appropriate $W_{e m g}$ is an issue involving the argument on how we can decide it, because normally $f^{*}$ is not a subject-specific estimation. For instance, when the literature based $F_{i s o}$ is taken for the elderly subject or athlete, the right term of the objective function can lead the inconsistency in the sense of subject-specific muscle strength. Thus, the identification of muscle strength is a trial to make the EMG-based muscle tension subject-specific and physically consistent. Even with the same choice of the $W_{\text {emg }}$, the solution $\boldsymbol{f}$ can be more reliable as its absolute value. In addition, the identified Muscle-Strength can be used for the performance index to evaluate the subject evolution in training and rehabilitation. In the optimistic scenario, software may automatically judge if the designated task can be performed by the patient who has particular motor function problem.

\section{EXPERIMENTAL RESULTS}

\section{A. Experimental setup}

The human motions are recorded by a commercial optical motion capture system consisting of 10 cameras (Motion Analysis). 35 reflective optical markers pasted on the body are captured by these cameras. We employed modified Helen Hayes Hospital marker set. They are located at the defined anatomical rigid points to diminish the influence from nonrigid skin and muscle movement to insure accuracy of inverse kinematics computations. The contact forces are measured by two force-plates (Kistler). EMG data are recorded at $1 \mathrm{kHz}$ with a 16-channel wireless EMG system (DELSYS) that is synchronized with other measurements. Bipolar surface electrodes are placed on the following 16 muscles for both legs; Gluteus Maximus, Rectus Femoris, Vastus Lateralis, long head of Biceps Femoris, Semimembranosus, Tibialis Anterior, medial head of Gastrocnemius, Soleus as shown in Fig.2.

First, subjects are requested to make Maximum Voluntary Contraction (MVC) for the extension and flexion in each joint of lower limbs. The corresponding EMG value was used as an initial reference for the normalization in EMG processing. Then, we record a T-pose of a few seconds for the geometric calibration. Next, a sequence of about 2 minutes of free exciting movements for the mass-distribution identification. For the better excitability for each joint DOF, subjects are requested to pay attention to articulate each body-segment. One session consisting of slow, normal and 


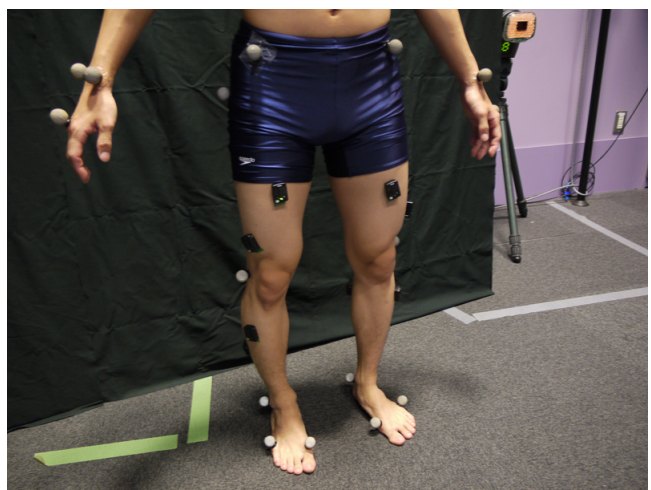

Fig. 2. Appearance of the experiment to measure EMGs and marker positions for lower limbs.

fast squat is repeated with different weighted loads ( $0 \mathrm{~kg}, 4 \mathrm{~kg}$, $8 \mathrm{~kg}$ ) around the waist.

\section{B. Identification of Mass-Distribution}

The identified geometric model is used to compute the inverse kinematics. Each joint information and the global coordinates are then used to compute the regressor matrix and the transformation matrix for the forces. The contact forces are measured by two force plates directly. The model of the body used for identification is simplified compared to the musculo-skeletal model. It consists in 34 degrees of freedom located between 15 links. Except for the upper torso, the links correspond to actual segment, thus it is not a simplification for lower limbs. The upper-torso is considered as one link for a precise identification, rather than using a decomposition similar to the musculo-skeletal model in several links of extremely small size, and thus with little mass and inertia, therefore difficult to identify precisely. The global parameters of the upper torso are then redistributed on the small links to match the specificity of the subject. The identified results are visualized graphically as shown in Fig. 3. The green color indicates that the inertial parameters are well identified, based on statistic evaluation. The figure in the link is the identified mass in kilograms. The results are consistent with expected values: no aberrant value, no negative value, a good right/left balance. And there is a clear difference in the identified Mass-Distribution between candidate A and candidate B, which are of different morphologies.

\section{Identification of Muscle-Strength}

In this first trial of Muscle-Strength (M-S) identification, we focused on knee joint torque variation under squat motions. First, we needed to extract motions which have torque variation well correlated to EMG-measured synergist muscle groups to be applied in Eq.16. The example of muscle activation transition is shown in the up of Fig.4. It was obtained from a squat motion with $8 \mathrm{~kg}$ load. Correlation analysis in the level of the net contribution matrix was performed to judge which EMG-measured synergist muscle group have enough correlation and contribute to the torque production. The knee element of contribution matrix in Rectus Femoris, Vastus Lateralis had significant correlation

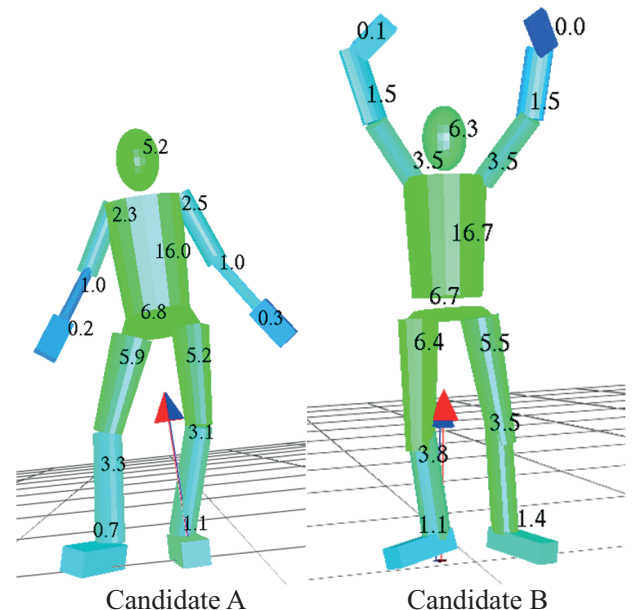

Fig. 3. The results of the identification for the two candidates. The figure represents the identified mass of each link in $\mathrm{kg}$ unit, the green color shows link which parameters are identified with excellent accuracy, the blue color shows link which parameters are small. The red arrow shows the direct force plate measurement, the blue arrow is the reconstruction using the identified parameters.
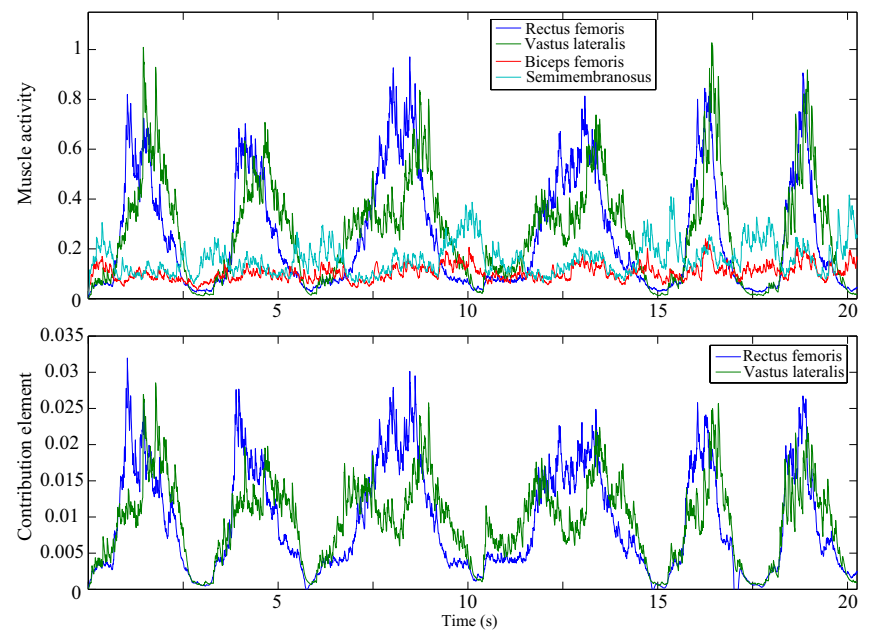

Fig. 4. The example of the muscle activation transition from a squat motion with $8 \mathrm{~kg}$ load (up). The knee element transition of contribution matrix in Rectus Femoris, Vastus Lateralis during the same motion. They showed significant correlation with knee joint torque during the motion (bottom)

as shown in the bottom of Fig.4. The squat motion with load made the situation where the knee extensor is required to be activated principally even in the knee bending phase. For Vastus Lateralis, the synergist muscle group: Vastus Medialis and Vastus Interioris are assumed to have same muscle activity. $F_{\text {iso }}$ was identified applying linear regression in Eq.16.

The estimation of muscle tensions was performed with the optimization as in Eq.17. The muscle force estimation with different weights $W_{e m g}$ was carried out with original settings and M-S identified settings for $F_{i s o}$ of Rectus Femoris and Vastus muscle group. The average error of left knee joint in the term of $\left|\boldsymbol{\tau}-\boldsymbol{J}^{T} \boldsymbol{f}\right|$ and the maximal estimated force in left Rectus Femoris of Candidate B during squat with 
$8 \mathrm{~kg}$ were evaluated as in Table I. The maximal estimated force indicates the force scale solved in the optimization. $F_{\text {iso }}$ of Rectus Femoris with original model was $1 k N . F_{\text {iso }}$ was identified as $685 N$ in Candidate B. $F_{\text {iso }}$ was identified as $802 N$ in Candidate A. For reference, the parameter in Delp musculoskeletal table is $780 \mathrm{~N}$. Considering the difference of musculoskeletal geometrical path, it is well identified within similar range.

TABLE I

KNEE TORQUE ERROR AND ESTIMATED FORCE SCALE FOR RECTUS FEMORIS WITH DIFFERENT $W_{e m g}$ IN STATIC OPTIMIZATION

\begin{tabular}{cccccc}
\hline \multirow{2}{*}{$W_{\text {emg }}$} & \multicolumn{2}{c}{ with M-S Ident } & & \multicolumn{2}{c}{ no M-S Ident } \\
\cline { 2 - 3 } \cline { 5 - 6 } & $\tau$ error & max force & & $\tau$ error & max force \\
\hline \hline 1 & 1.1 & 632 & & 0.87 & 940 \\
$5 \times 10^{-1}$ & 0.99 & 626 & & 0.78 & 938 \\
$10^{-1}$ & 0.59 & 595 & & 0.49 & 871 \\
$5 \times 10^{-2}$ & 0.45 & 591 & & 0.39 & 832 \\
$10^{-2}$ & 0.26 & 585 & & 0.23 & 543 \\
$5 \times 10^{-3}$ & 0.23 & 580 & & 0.20 & 433 \\
$10^{-3}$ & 0.19 & 483 & & 0.17 & 337 \\
\hline
\end{tabular}

$\tau$ error showed similar result both for the optimization with/without M-S identification. The estimated force is less influenced by $W_{e m g}$ with M-S identification.

Normally when $W_{e m g}$ is increased, $\tau$ error can be increased because the solution needs to be found keeping physical consistency from ID and respecting EMG-based model output. $\tau$ error showed similar result both for the optimization with/without M-S identification. However, when M-S identification is not performed, the estimated force scale varied a lot depending on $W_{e m g}$. It is not preferable since the estimated force is influenced by the computation setting. It can be explained by the following: as the muscle reference force $f_{*}$ is not realistic, minimizing the right term in the objective function causes problem. Thus, the solution is easily influenced by the difference of $W_{e m g}$. In addition, as $F_{i s o}$ is $1 k N$ for original model, we can see the solution without M-S Identification is misled while increasing $W_{\text {emg }}$. Thus, the estimated value is still usable as the comparative study with other muscles such as distribution ratio, but not for the individual analysis with different tasks. For very small $W_{e m g}$, even in M-S identified case, it started to give different solution, but it is normal as it starts not to respect to EMG-based model output. In any case, the optimal setting of $W_{e m g}$ and $W_{d y n}$ is hard to be predicted before the analysis. Since the solution with M-S identification became stable with much larger range of $W_{e m g}$, this first results highlight the effectiveness of Muscle-Strength identification.

Next, the identified model was applied for the muscle forces estimation in another squat motions with $4 \mathrm{~kg}$ load. Fig. 5 shows the estimated muscle forces of Rectus Femoris, Vastus Lateralis and Vastus Medialis with different speeds (first two series are normal speed, second two are slow and last two are fast). The corresponding visualizations of estimated muscle tensions at the indicated time instant are depicted in the bottom.

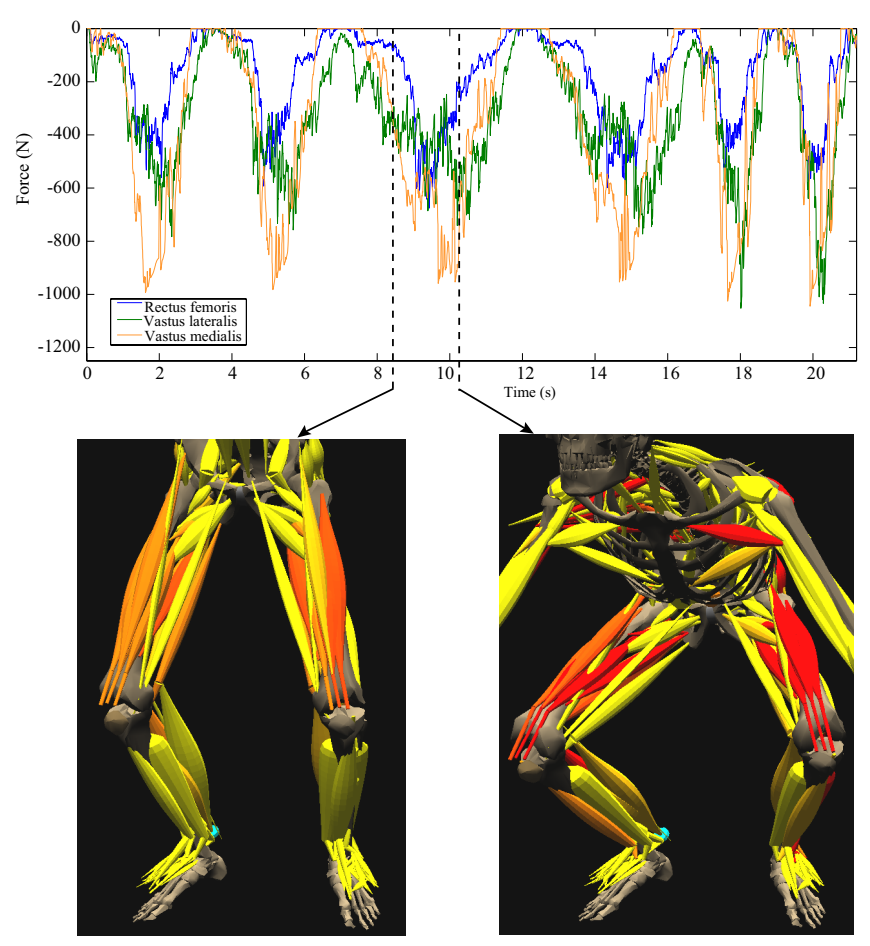

Fig. 5. Estimated muscle forces of Rectus Femoris, Vastus Lateralis and Vastus Medialis using the identified model in squat motion with $4 \mathrm{~kg}$ load with different speeds (first two series are normal speed, second two are slow and last two are fast) (up). The corresponding visualization of estimated muscle tensions at the indicated time instant (bottom).

\section{CONCLUSION}

In this paper we have presented a method that allows to reliable subject-specific muscle forces estimation with Muscle Strength and Mass Distribution Identification. The proposed method features:

- Mass Distribution Identification to improve the joint torque estimation accuracy,

- Muscle Strength Identification to improve the muscle force estimation accuracy,

- The above features result in subject-specific musculoskeletal dynamics computation to compensate the drawback of conventional computer aided analysis such as the assumptions in body-segment parameters and muscle strength parameters.

The result as in Fig. 5 indicated the starting timing of contractions of knee extensor muscles is visualized in quite reasonable way and the estimated value is expected to be reliable since this model computation is considering the subject-specific mass distribution and subject-specific muscle strength of knee extensors along with EMG activities of surface muscles and all the inner muscles contributions in the optimization process.

However, in order to apply the proposed method to all the other joints, we still have open problem such as how we can automatically detect the motions which have high correlation between torque variation and net contribution matrix. The excitation index tracking algorithm in torquemuscle force mapping would be necessary in order to make 
this framework applicable in systematic way. The knowledge about modular control in synergist muscle group [19] is recently well accepted in neuroscience. It would help a lot to decrease the actuation redundancy problem. Muscle grouping to realize real-time computation is already implemented in [20]. Muscle grouping concept would help also for systematical detection of high correlated motion in torque-muscle force mapping.

Even though the above problems are required to be solved, the proposed framework toward subject-specific musculoskeletal modeling would contribute to a patientoriented computational rehabilitation. In addition to subjectspecificity, human characteristics are basically time variant, for instance, neuromuscular dynamics may vary according to muscle fatigue. In order to correspond to such time-varying characteristic, a model-based approach which is combined with adaptive identification would enable to bring a new modality in musculoskeletal modeling as in [21].

\section{ACKNOWLEDGMENTS}

The authors acknowledge the use of $s D I M S$, multibody and musculoskeletal dynamics computation software [4][5], developed in the University of Tokyo.

\section{REFERENCES}

[1] T.A. McMahon, "Muscles, Reflexes, and Locomotion", Princeton University Press, 1984.

[2] S.L. Delp and J.P. J. P. Loan, "A computational framework for simulating and analyzing human and animal movement", IEEE Computing in Science and Engineering, vol.2, pp. 46-55, 2000.

[3] S.L. Delp, F.C. Anderson et al, "OpenSim: Open-Source Software to Create and Analyze Dynamic Simulations of Movement", IEEE Trans. on Biomedical Engineering, vol. 54, no. 11, pp 1940-1950, 2007.

[4] Y. Nakamura, K. Yamane, Y. Fujita, and I. Suzuki, "Somatosensory Computation for Man-Machine Interface From Motion-Capture Data and Musculoskeletal Human Model", IEEE Trans. on Robotics, vol. 21, no. 1, pp 58-66, 2005.

[5] K. Yamane, Y. Fujita, and Y. Nakamura, "Estimation of physically and physiologically valid somatosensory information," in Proc. of the IEEE Int. Conf. on Robotics and Automation, Barcelona, Spain, 2005, pp. 2635-2641.

[6] P. de Leva, "Adjustments to Zatsiorsky-Seluyanov's segment inertia parameters", J. Biomech, 29(9), S. pp.1223-1230, 1996.

[7] T.S. Buchanan, D.G. Lloyd, K. Manal and T.F. Bessier, "Neuromusculoskeletal modeling: estimation of muscle forces and joint moments and movements from measurements of neural command", Journal of Applied Biomechanics, no. 20, pp. 367-395, 2004.

[8] G. Venture, K. Ayusawa, and Y. Nakamura, "Real-time identification and visualization of human segment parameters," in Proc. of the IEEE Int. Conf. on Engineering in Medicine and Biology, 2009, pp. 39833986.

[9] K. Ayusawa, G. Venture, and Y. Nakamura, "Real-time implementation of physically consistent identification of human body segments," in Proc. IEEE Int. Conf. on Robotics and Automation (to be published), 2011.

[10] D.G. Lloyd and T.F. Bessier, "An emg-driven musculoskeletal model to estimate muscle forces and knee joint moment in vivo", Journal of Biomechanics, no. 36, pp. 765-776, 2003.

[11] A.V. Hill, "The heat of shortening and the dynamic constants in muscle", Proceeding of the royal society, London, Sre. B, vol. 126, pp. 136-195, 1938.

[12] F.E. Zajac "Muscle and tendon: properties, models, scaling and application to biomechanics and motor control". CRC Critic. Rev. in Biomed. Eng. 17: 359-411, 1989.

[13] J. Potvin, R. Norman, and S. McGill, gMechanically corrected EMG for the continuous estimation of erector spinae muscle loading during repetivtive lifting,h European Journal of Applied Physiology, vol. 74, pp. 119-132, 1996.
[14] S. Stroeve, "Impedance Characteristics of a Neuro-Musculoskeletal Model of the Human Arm I: Posture Control", Journal of Biological Cybernetics, vol. 81, pp. 475-494, 1999.

[15] M. Hayashibe, M. Benoussaad, D. Guiraud, P. Poignet, C. Fattal, "Nonlinear Identification Method Corresponding to Muscle Property Variation in FES - Experiments in Paraplegic Patients," in Proc. of the IEEE/RAS-EMBS Int. Conf. on Biomedical Robotics and Biomechatronics, 2010, pp. 401-406.

[16] M. Sartori, M. Reggiani, D.G. Lloyd, and E. Pagello, "An EMG-driven Musculoskeletal Model of the Human Lower Limb for the Estimation of Muscle Forces and Moments at the Hip, Knee and Ankle Joints in vivo," in Proc. of Int. Conf. on Simulation, Modeling and Programming for Autonomous Robots, 2010, pp. 137-146.

[17] K. Yoshida, D.N. Nenchev, and M. Uchiyama, "Moving base robotics and reaction management control," in Proc. of the Seventh Int. Symp. of Robotics Research, 1995, pp. 100-109.

[18] W. Khalil and E. Dombre, Modeling, identification and control of robots, Hermès Penton, London-U.K, 2002.

[19] Y. P. Ivanenko G. Cappellini, N. Dominici, R. E. Poppele, and F. Lacquaniti, "Modular Control of Limb Movements during Human Locomotion,” in J. Neurosci, vol. 27, no. 41, 2007, pp 11149-11161.

[20] A. Murai, K. Kurosaki, K. Yamane, and Y. Nakamura, "Computationally Fast Estimation of Muscle Tension for Realtime Bio-feedback," in Proc. of the IEEE Int. Conf. on Engineering in Medicine and Biology, 2009, pp. 6546-6549.

[21] Q. Zhang, M. Hayashibe, P. Fraisse, D. Guiraud, 'FES-Induced Torque Prediction with Evoked EMG Sensing for Muscle Fatigue Tracking", IEEE/ASME Transactions on Mechatronics, Special Issue on Sensing Technologies for Biomechatronics, (in print), 2011. 